
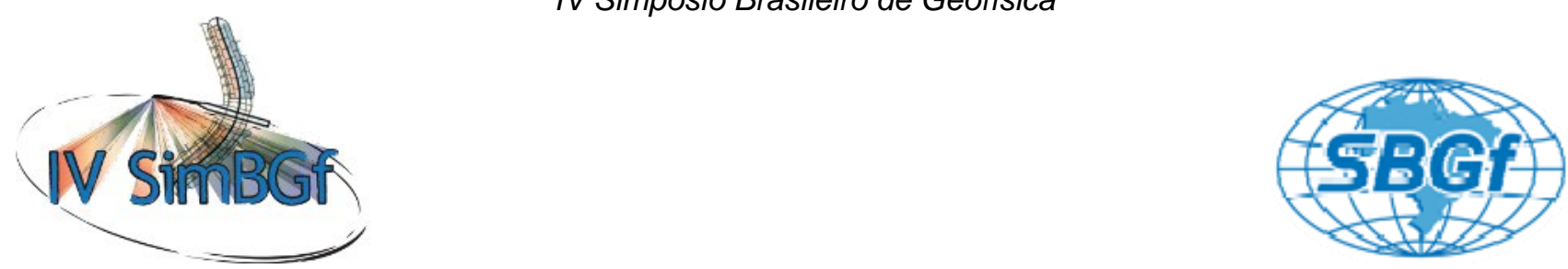

\title{
Caracterização geotécnica de solos marinhos empregando atributos derivados da Sísmica: a importância da incorporação da informação elástica
}

Jorge Fiori Fernandes Sobreira (*), PETROBRAS

Marlos Lipski, PETROBRAS

Anderson Luiz Pimentel, PETROBRAS

Luciano Arêas Carvalho, Schlumberger

Fredy Alex Villaorduna Artola, Schlumberger

Evelin Márquez, Schlumberger

Copyright 2008, SBGf - Sociedade Brasileira de Geofísica

Este texto foi preparado para a apresentação no IV Simpósio Brasileiro de Geofísica, Belém, 14 a 17 de novembro de 2010. Seu conteúdo foi revisado pelo Comitê Técnico do IV SimBGf, mas não necessariamente representa a opinião da SBGf ou de seus associados. É proibida a reprodução total ou parcial deste material para propósitos comerciais sem prévia autorização da SBGf.

\begin{abstract}
Geotechnical characterization of soils is a critical step whenever major on land Engineering enterprises, such as dams or tunnels need to be planned. While this task can be more easily and cheaply undertaken onshore, in the case of marine infrastructure offshore, such as pipelines or rigs for the Oil Industry, an expensive campaign of geotechnical boreholes for direct and high resolution testing and sampling of marine soil becomes necessary. However, information thus obtained is rather local regarding its spatial significance. Deriving information embedded with geotechnical significance indirectly, from geophysical data such as seismic, can be a much cheaper alternative, and this paper shows how this unconventional approach was evaluated. A further motivation of using seismic data relies on that these are able to provide information whose spatial significance is rather areal or regional, which allows extrapolating the known geotechnical behavior between boreholes in a more calibrated way, and thus more safely. This approach was investigated and applied for the near seabed section of two offshore oil fields in Campos Basin, Eastern Brazilian Margin: Marlim and Albacora fields. In this approach it's emphasized the importance of incorporating elastic information in order to obtain more robust results from seismic data.
\end{abstract}

\section{Introdução}

O emprego de dados sísmicos em aplicações para fins de Geotecnia não chega a constituir novidade. Em especial, as ondas superficiais (denominadas ondas Scholte no caso da interface líquido-sólido, como o fundo do mar) são especialmente úteis para a extração de propriedades da seção rasa na qual elas se propagam. No entanto, este tipo de onda tende a não estar representado em registros sísmicos marítimos em situações de águas profundas, justamente onde reside o interesse principal da Indústria de Óleo no Brasil. Assim, uma abordagem baseada apenas em dados sísmicos das chamadas "ondas de corpo" ou "ondas de volume" (isto é, onda $\mathrm{P}$ ou compressional e, eventualmente, onda $\mathrm{S}$ ou cisalhante), em geral disponíveis nas várias áreas (e por vezes de excelente qualidade, embora normalmente não processados de maneira dedicada para a seção mais rasa), foi aqui empreendida.

Seguindo a linha dos trabalhos anteriores de Sobreira et al. (2009, 2010), nos quais se discute a extração de atributos com significado geotécnico derivados da inversão sísmica, este trabalho procura demonstrar a importância da incorporação da informação elástica ao processo, seja através da inversão simultânea de vários subconjuntos de empilhamentos parciais (de onda PP), ou, mais desejavelmente, através da incorporação do modo de onda PS, obtido de dados sísmicos multicomponentes (inversão conjunta).

\section{Problema Investigado}

Uma típica campanha geotécnica marítima consiste da perfuração de diversos furos geotécnicos, rasos, através dos quais parâmetros como o chamado Su (undrained shear strength) podem ser obtidos. Este pode ser considerado um parâmetro geotécnico direto, relacionado à resistência in situ dos sedimentos à penetração de um dispositivo conhecido como cone-penetrômetro (ou CPT). Adicionalmente, medidas diretas de densidade, e em alguns casos, da velocidade cisalhante (Vs), são também obtidos a partir destes furos. Atributos derivados de dados sísmicos, como é o caso dos módulos elásticos, podem ser extraídos e comparados com aqueles parâmetros diretos, sempre tendo em mente que estes atributos dizem respeito não ä resistência à deformação em si, mas ao stiffness ou grau de rigidez dos sedimentos (embora ambas as grandezas se relacionem à mesma dimensão física, que vem a ser a pressão). Além disto, enquanto a onda sísmica perturba o meio no qual se propaga de maneira elástica, o ensaio geotécnico implica um regime rúptil, onde ocorre efetivamente a ruptura dos sedimentos, e no qual o nível de deformação é algumas ordens de grandeza mais elevado. Ainda que reconhecidas estas aparentes limitações intrínsecas, foi decidido testar-se a abordagem baseada em dados 
sísmicos para a extração de atributos com significado geotécnico.

\section{Método empregado}

A abordagem de extração de atributos "pseudogeotécnicos" com base em dados sísmicos foi fundamentada na inversão sísmica concomitante de subconjuntos de dados de empilhamento parcial, por faixas de ângulo (inversão simultânea), cujo algoritmo emprega uma modificação da aproximação da refletividade de Aki e Richards (1980), com otimização global. Tanto dados sísmicos de onda $\mathrm{P}$ quanto de onda PP e PS (inversão conjunta) foram empregados para esta inversão simultânea.

\section{Dados utilizados}

Os dados sísmicos utilizados consistem de: a) dados sísmicos 3D de alta resolução, de onda $P$ apenas, adquiridos com streamer na região do campo de Marlim, na Bacia de Campos; e b) dados sísmicos 2D/ 4C (4 componentes), de ondas PP e PS, adquiridos com cabos de fundo oceânico (OBC's) na região do campo de Albacora, também na Bacia de Campos.

Os furos geotécnicos, além da informação geotécnica direta (Su), a ser comparada com aquela derivada da Sísmica, também forneceram dados de densidade e de Vs. A informação de Vp foi derivada dos dados in situ de Vs por meio da equação de mudrock (Castagna et al., 1985), escolhida após testarem-se várias outras relações matemáticas. Estes dados de $\mathrm{Vp}$, Vs e densidade também permitiram construir logs na posição dos furos, empregados tanto para amarração quanto para a geração do modelo de baixa freqüência, requerido pelo processo de inversão.

Horizontes-chave rasos, incluindo o fundo do mar, foram ainda interpretados tanto nos dados de onda $P$ (3D de Marlim, exemplificado na figura 1), como nos dados 2D de onda PP e PS (em suas respectivas escalas de tempo sísmico originais) de Albacora. Neste último caso, os horizontes interpretados nos dois conjuntos de dados foram tentativamente correlacionados a interfaces geológicas comuns. Estes horizontes proveram guia estrutural ao processo de inversão sísmica.

\section{Resultados - Inversão sísmica simultânea de dados PP}

A partir das propriedades derivadas diretamente da inversão sísmica simultânea, em função da incorporação de informação com conteúdo elástico (impedância acústica, impedância cisalhante e densidade), foram produzidos, por manipulação algébrica entre estes, módulos elásticos (dinâmicos) tais como o módulo de cisalhamento e o coeficiente de Lamé (conhecidos conjuntamente como parâmetros de Lamé), os quais, por sua vez, foram combinados entre si e com a densidade para compor outros atributos. Adicionalmente, outros módulos como o módulo de Young e o módulo de bulk (ou de incompressibilidade), de interesse corriqueiro em problemas de Engenharia, foram produzidos, além das próprias velocidades intervalares, $\mathrm{Vp}$ e Vs.

Exemplos de mapas de atributos sísmicos derivados da inversão sísmica simultânea 3D na área de Marlim (figuras 2 e 3) mostram como atributos relacionados à onda $\mathrm{S}$, como o módulo de Young (também expresso em unidades de pressão) tendem a reproduzir melhor (ao menos em termos qualitativos) o comportamento geotécnico esperado ou conhecido, do que aqueles derivados essencialmente da onda $\mathrm{P}$, como o módulo de

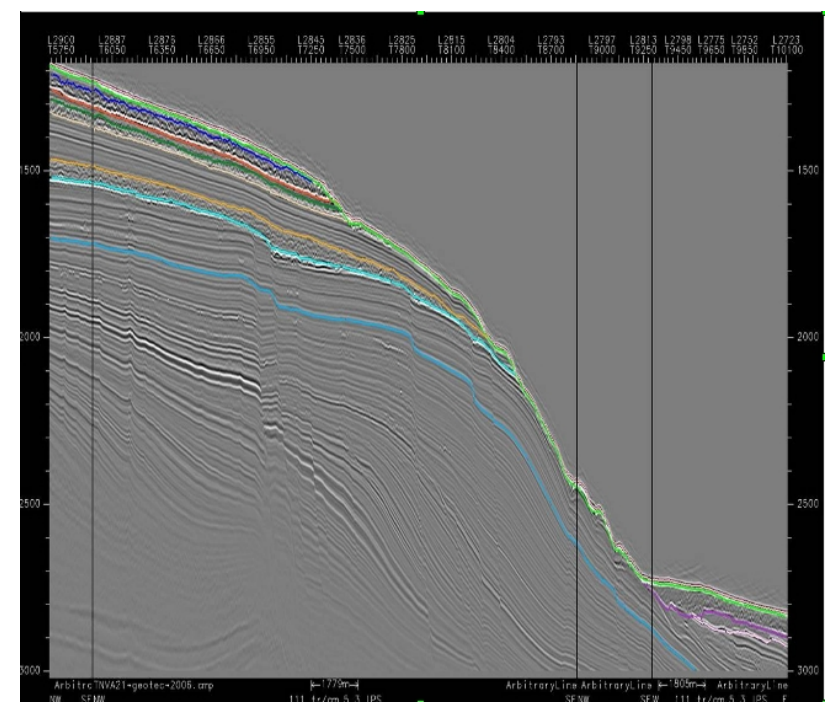

Figura 1 - Seção sísmica representativa, orientada NWSE, na região do campo de Marlim, com horizontes rasos interpretados, assinalados em diferentes cores. Escala vertical em tempo sísmico (duplo) variando de 1.200 a 3.000 ms. A extensão horizontal da seção é de cerca de $20 \mathrm{~km}$, e a declividade do fundo do mar, com exagero aparente na Sísmica, não chega a $10^{\circ}$

incompressibilidade. Estes mapas dizem respeito à reflexão mais rasa dos volumes sísmicos invertidos, a qual, de acordo com estimativas locais, é tida como aproximadamente representativa do comportamento médio dos primeiros 15 a $20 \mathrm{~m}$ de sedimentos abaixo do fundo do mar. Outro atributo, relacionado à onda $\mathrm{S}$, que vem a ser o módulo de cisalhamento, também apresenta correlação razoável com o comportamento geotécnico conhecido, o que é exemplificado na figura 4, que também mostra que os principais contrastes do perfil de Su de um furo geotécnico localizado no pé do talude continental da região de Marlim - onde importante variação geotécnica ocorre na seção mais rasa - tendem a ser reproduzidos (ao menos em termos qualitativos).

De uma maneira geral (comparando-se com a figura 1), observa-se que os maiores valores dos módulos elásticos dinâmicos tendem a ocorrer para sedimentos rasos mais antigos (e neste caso também mais rígidos ou consolidados), dominantes no compartimento intermediário da área, enquanto que, por outro lado, menores valores desses módulos tendem a coincidir com 
a zona de ocorrência de sedimentos rasos mais jovens (e neste caso, também menos consolidados), dominantes nos compartimentos proximal (de lâmina d’água mais rasa) e distal (de lâmina d’água mais profunda) da área.

Com base no relacionamento acima observado, uma relação matemática entre parâmetros geotécnicos diretos (como Su) e módulos elásticos dinâmicos, derivados da Sísmica, não analítica, mas empírica, e assim de validade antes local do que genérica, pode assim ser estabelecida. Na região de Marlim, esta relação, positiva, pode ser considerada válida para a maior parte dos sedimentos, como é o caso dos siliciclásticos com estrutura interna "bem comportada", mas não necessariamente para outros tipos, como sedimentos carbonáticos/ coralíneos ou mesmo siliciclásticos com estrutura interna caótica ou desorganizada. Este ponto, embora de interesse geral para a investigação, não constitui o escopo deste trabalho, razão pela qual não será aqui detalhada.

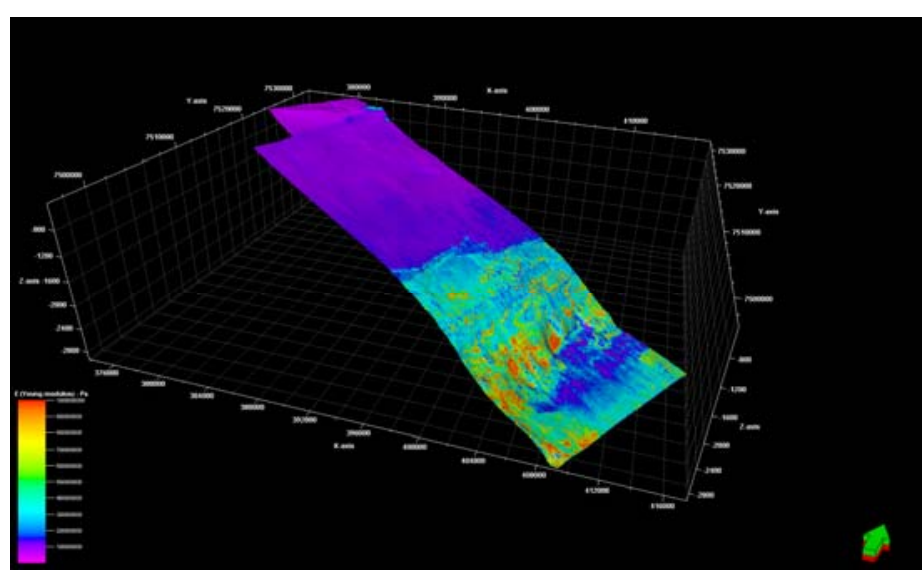

Figura 2 - Módulo de Young para a área de Marlim, com relevo do fundo do mar sobreposto. A escala de cores varia de $1,0 \times 10^{8}$ (magenta) a $1,0 \times 10^{9}$ (vermelho) Pa..Comparar com a figura 1.

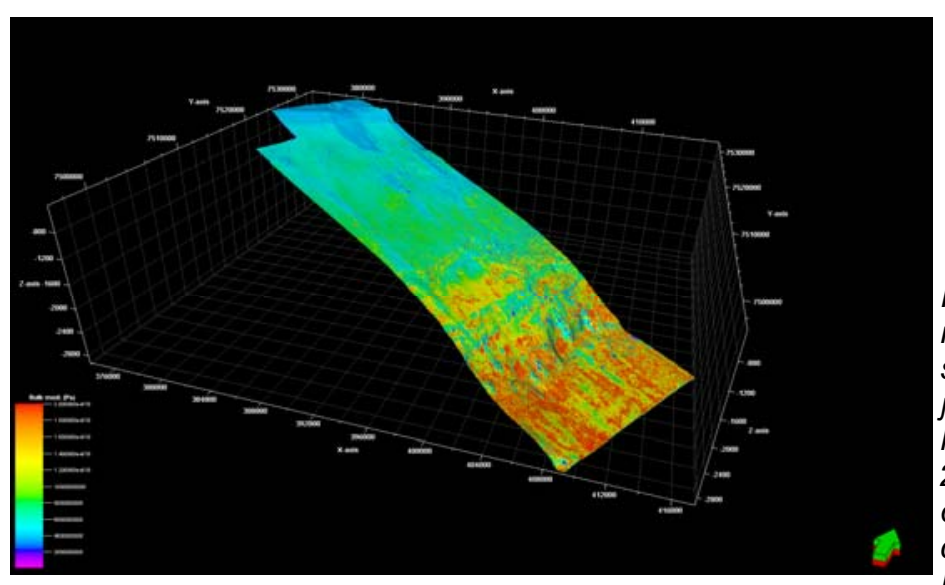

Figura 3 - Módulo de incompressibilidade para a área de Marlim, com relevo do fundo do mar sobreposto. A escala de cores varia de $2,0 \times 10^{9}$ (magenta) a 2,0 $010^{10}$ (vermelho) $\mathrm{Pa}$. Comparar com a figura 1

Apenas para acrescentar, Sobreira et al. (2010) obtiveram resultados qualitativamente semelhantes a esta abordagem baseada na inversão sísmica simultânea (embora considerados menos robustos) com base na mais direta (e mais expedita) análise da refletividade AVO, mais precisamente, a amplitude (far - near, normalizada) do refletor correspondente ao fundo do mar.

\section{Resultados - Inversão sísmica simultânea e conjunta (de dados PP e PS)}

A disponibilidade de dados sísmicos multicomponentes (2D/ 4C) na região do campo de Albacora, onde também estão disponíveis furos geotécnicos com informação de Vs obtida in situ, permitiu empreender-se uma inversão simultânea e também conjunta (isto é, empregando tanto subconjuntos de empilhamentos parciais de dados de onda PP quanto de onda PS). Os mesmos atributos produzidos no caso da inversão sísmica 3D foram também aqui gerados, agora a partir de dados sísmicos multicomponentes. Da mesma forma que no caso do emprego de dados de onda $P$ apenas, constatou-se que atributos relacionados à onda $\mathrm{S}$, como o módulo de cisalhamento, mostram-se especialmente sensíveis às variações geotécnicas conhecidas. Esta constatação é exemplificada na figura 5, onde são comparados os traços invertidos, na posição de dois furos geotécnicos, que contêm informação in situ de Vs e densidade, com os logs destes mesmos furos. Esses atributos, derivados por manipulação algébrica a partir dos resultados diretos da inversão sísmica (neste caso, inversão conjunta), são comparados com os resultados da inversão simultânea utilizando apenas de dados da onda P. É notável como o módulo de cisalhamento é capaz de reproduzir de modo

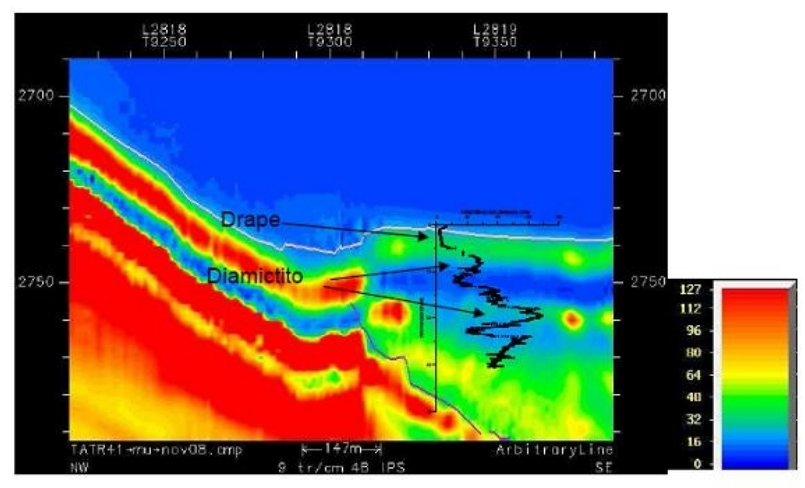

Figura 4 - Detalhe da seção do módulo de cisalhamento na região da base do talude em Marlim, com perfil superposto de Su de um furo geotécnico próximo. A janela de Su varia de 0 a $30 \mathrm{~m}$ (na vertical) e de 0 a 160 $\mathrm{kPa}$ (na horizontal), e a janela sísmica varia de 2.690 a $2.790 \mathrm{~ms}$ (tempo duplo) na vertical, e tem cerca de $1 \mathrm{~km}$ de extensão horizontal. A escala de cores do módulo de cisalhamento varia de 0 (azul escuro) a 127 (vermelho) $\mathrm{MPa}$. "Drape" refere-se à cobertura sedimentar hemipelágica (inconsolidada) e "diamictito" refere-se a sedimentos menos jovens, relacionados a fluxos de detritos. Comparar com a figura 1.

razoável o comportamento geotécnico esperado, e, inclusive, representar detalhes da seção sedimentar rasa 
(tais como camadas delgadas). Igualmente notável é o valor agregado aos resultados pela incorporação de dados de onda PS (graças à disponibilidade de dados sísmicos multicomponentes nesta área em especial).

A figura 6 apresenta os resultados para outros módulos elásticos derivados da Inversão Sísmica, como o módulo de Young e o módulo de incompressibilidade, para um dos furos geotécnicos da área. Estes resultados reforçam a melhor representação do comportamento geotécnico por atributos sísmicos relacionados à onda $\mathrm{S}$ (caso do módulo de Young) em relação a atributos relacionados unicamente à onda $\mathrm{P}$ (caso do módulo de incompressibilidade). Nesta mesma figura pode-se observar que, se por um lado a inclusão de dados de onda PS não chega a contribuir para melhorar o resultado para atributos como o módulo de incompressibilidade, por outro, melhora significativamente os resultados de atributos como o módulo de Young (ou o próprio módulo de cisalhamento, tal como ilustrado na figura 5).

\section{Discussão}

A relação entre atributos sísmicos (módulos dinâmicos) e propriedades ou parâmetros geotécnicos de solos ou sedimentos rasos tem sido objeto de uma série de trabalhos anteriores, embora essencialmente voltados para aplicações em terra. Dentre estes, pode-se destacar Suyama et al. (1983), que demonstram que Vs, mais do que $\mathrm{Vp}$, mostra-se especialmente sensível à variação de um determinado parâmetro geotécnico por estes autores tomado como referência (além do plote cruzado com este parâmetro também apresentar menor dispersão para Vs do que para $V p$ ), o que vem a corroborar os resultados aqui obtidos, que sugerem que atributos sísmicos tendem a apresentar correlação positiva com parâmetros elásticos, sendo esta correlação mais evidente para o caso dos atributos sísmicos derivados da onda $\mathrm{S}$, tais como o módulo de cisalhamento e o módulo de Young.

\section{Conclusões}

A abordagem sísmica, baseada na extração de atributos elásticos com significado geotécnico através de inversão (simultânea) de dados sísmicos, conduziu a resultados com razoável correlação qualitativa com a informação geotécnica conhecida ou esperada, não obstante diferenças intrínsecas importantes, existentes com relação ao experimento geotécnico, direto. A obtenção de estimativas mais quantitativas, de maior interesse para a Engenharia, constitui o escopo de trabalho ora em curso.

O emprego da inversão sísmica baseada na análise de AVO foi demonstrado para aplicações nãoconvencionais, focadas em objetivos incomumente rasos, relacionados a rochas pobremente consolidadas.

A incorporação da informação elástica, a partir da utilização de vários subconjuntos de empilhamentos parciais do dado sísmico (inversão simultânea), seja ele apenas de onda $\mathrm{P}$, ou preferencialmente incluindo também dados sísmicos PS (ou SS, se disponíveis), agrega robustez aos resultados da inversão sísmica, permitindo aprimorar-se a capacidade ou o potencial de
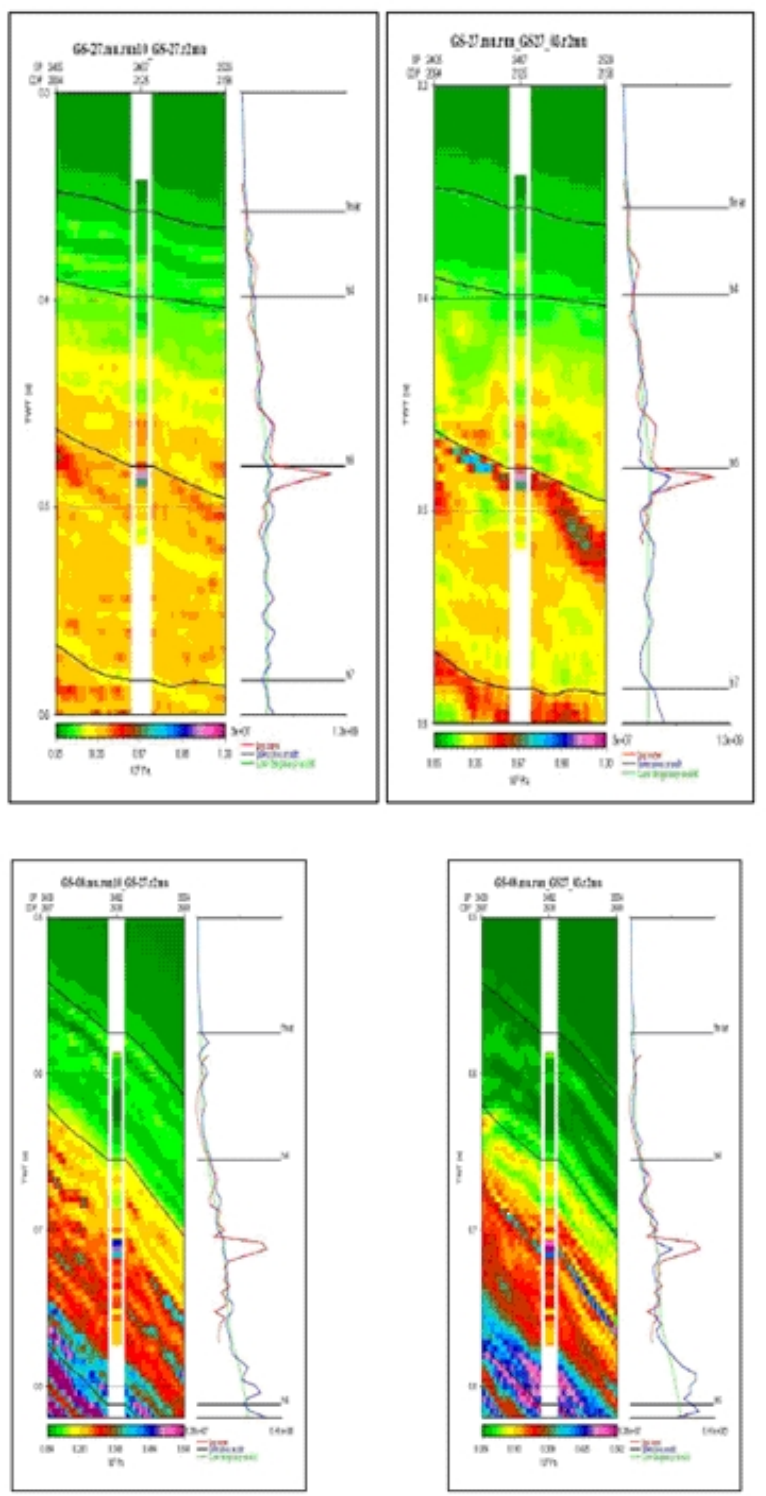

Figura 5 - Comparação do traço invertido (em cor azul) e do log (em cor vermelha) do módulo de cisalhamento em dois furos geotécnicos da área de Albacora, o GS-27 (acima) e o GS-08 (abaixo); também indicado (em cor verde) está o traço do correspondente modelo de baixa frequencia utilizado. Para cada um dos furos, o painel da esquerda refere-se aos resultados da inversão sísmica simultânea empregando apenas a onda $P$, enquanto $o$ painel da direita refere-se aos resultados da inversão sísmica simultânea e também conjunta, isto é, empregando tanto dados de onda $P$ quanto $P S$. A escala de cores para o trecho da seção de módulo de cisalhamento em torno dos furos geotécnicos varia de 0,05 (verde) a 1,30 (magenta) GPa para os painéis superiores, e de 0,064 (verde) a 0,641 (magenta) GPa para os painéis inferiores. 
predição do comportamento geotécnico da seçãoobjetivo. Esta informação elástica é especialmente útil no caso da caracterização da rigidez ou grau de consolidação dos sedimentos, ao focar no efeito do arcabouço da rocha em si. $\mathrm{E}$ as propriedades deste arcabouço são aquelas que efetivamente controlam as propriedades geotécnicas ou mecânicas (estáticas), em detrimento de outros efeitos, como o conteúdo dos poros.
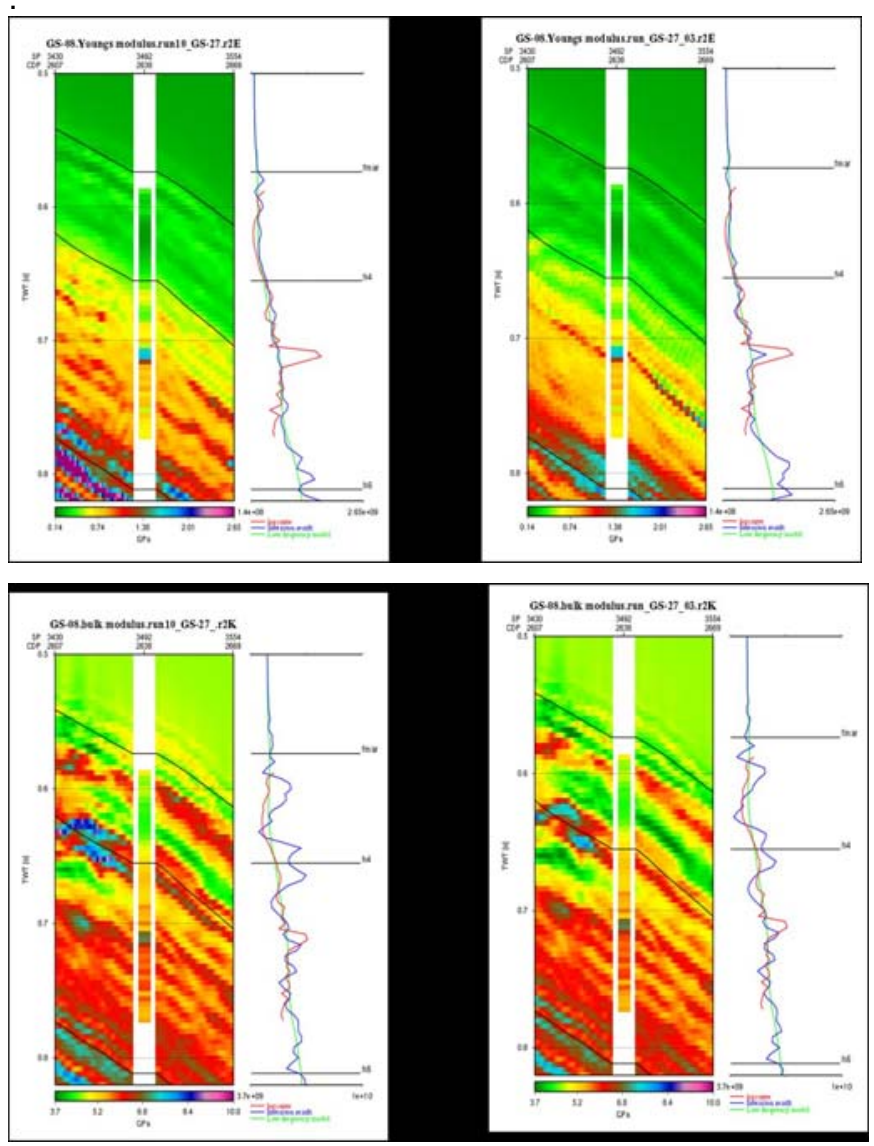

Figura 6 - Comparação do traço invertido (em cor azul) e do log (em cor vermelha) do módulo de Young (acima) e do módulo de incompressibilidade (abaixo) no furo geotécnico GS-08, da área de Albacora; também indicado (em cor verde) está o traço do correspondente modelo de baixa freqüência utilizado. Para cada um dos módulos, o painel da esquerda refere-se aos resultados da inversão sísmica simultânea empregando apenas a onda $P$, enquanto o painel da direita refere-se aos resultados da inversão sísmica simultânea e também conjunta, isto é, empregando tanto dados de onda $P$ quanto PS. A escala de cores para o trecho da seção em torno do furo geotécnico varia de 0,14 (verde) a 2,65 (magenta) GPa para os painéis superiores (módulo de Young), e de 3,7 (verde) a 10,0 (magenta) GPa para os painéis inferiores (módulo de incompressibilidade).

\section{Agradecimentos}

Gostaríamos de agradecer à PETROBRAS por ter autorizado a divulgação deste trabalho.

\section{Referências}

Aki, K., Richards, P. G., 1980. Quantitative Seismology Theory and methods, vol. 1, W. H. Freeman and Company.

Castagna, J., Batzle, M., Eastwood, R. L.., 1985. Relationship between compressional and shear wave velocities in clastic silicate rocks. Geophysics, 50, 551557.

Sobreira, J. F. F., Lipski, M., Carvalho, L. A., Márquez, E., 2009. Simultaneous seismic inversion applied to geotechnical analysis of near seabed sediments. Proceedings, $11^{\text {th }}$ International Congress of the Brazilian Geophysical Society, Salvador.

Sobreira, J. F. F., Lipski, M., Carvalho, L. A., Márquez, E., 2010. Geotechnical characterization based on seismic data approaches applied in Campos Basin, Southeastern Brazilian margin. The Leading Edge, vol. 29, n. 7, p. 842846.

Suyama, K., Imai, T., Ohtorno, H., Ohta, K., Ohishi, Y., 1983. Shallow seismic reflection exploration in soft ground using SH waves. SEG expanded abstracts 2, p. 103-105, $53^{\text {rd }}$ Ann. Intern. Mtg. of the SEG, Las Vegas. 